\title{
Estimates of Heterosis for Seed Yield and its Contributing Characters in Opium Poppy (Papaver somniferum L.)
}

\author{
K. Nesara ${ }^{1 *}$, Amit Dadheech ${ }^{1}$, N.K. Padiwal ${ }^{1}$, S. Bhaskar Reddy ${ }^{2}$ and Rajwanti Saran ${ }^{3}$ \\ ${ }^{1}$ Department of Genetics and Plant breeding, Rajasthan College of Agriculture, MPUAT, \\ Udaipur-313 001 (Rajasthan), ${ }^{2}$ College of Agriculture (Kerala Agriculture University) \\ Vellayani, ${ }^{3}$ Rajasthan Agricultural Research Institute (SKNAU), Durgapura, Jaipur, India \\ *Corresponding author
}

\section{A B S T R A C T}

Line $\times$ Tester analysis was carried out using 13 lines and 3 testers to estimate heterosis on seed yield and its contributing characters in Opium Poppy. The 39 resulting hybrids along

\section{Keywords}

Heterosis,

Heterobeltosis,

Economic heterosis,

Line $\times$ Tester,

Opium poppy

Article Info

Accepted:

18 August 2020

Available Online:

10 September 2020 with their parents were evaluated in Randomized Block Design during Rabi 2019-2020.

The analysis of variance revealed the presence of variability among parents and crosses for the characters studied. Significant parents v/s hybrids contrast for all characters, except for days to 50 percent flowering, days to maturity and number of leaves per plant, indicated the presence of considerable amount of genetic diversity in the material tested. The experimental results for seed yield revealed that out of 39 hybrids, 15 hybrids recorded significant and positive relative heterosis, 11 and one hybrid recorded positive significant heterobeltosis and economic heterosis, respectively. The maximum heterosis and heterobeltosis was observed in cross UOP-149 $\times$ UOP-79 for seed yield per plant, harvest index, capsule husk yield per plant and number of stigmatic rays per capsule, cross UOP$68 \times$ UOP-20 for seed yield and harvest index and cross UOP-64 $\times$ UOP-20 for seed yield per plant, latex yield per plant and harvest index for seed yield. UOP-128 $\times$ UOP-20 was the only cross which exhibited maximum heterosis, heterobeltosis and economic heterosis for seed yield in desirable direction. Thus, this cross can be recommended for exploitation of heterosis and to obtain suitable segregants.

\section{Introduction}

Opium poppy (Papaver somniferum L.) is an ancient medicinal crop known to mankind. Papaver somniferum L. $(2 \mathrm{n}=22)$, is a flowering plant of family Papavaraceae. Poppy is generally considered to be self pollinating plant, but some studies have reported certain degree of outcrossing (Singh et al., 1999). The outcrossing is due to insect activity, especially bees. Morphine, coedine, thebaine, narcotine and papaverine are the major alkaloids which are synthesized in specialized cells called laticifers in mature capsule and used to treat almost all type of pains. India is one of the largest producers of opium alkaloids in the world. The Narcotics Department, Government of India issues annual renewable licenses to the farmers. 
Heterosis is superiority of the hybrid over the mid parent or better parent or standard variety and is result of allelic or non-allelic interaction of gene under the influence of particular environment. Estimation of heterosis over mid parent (relative heterosis) and over better parent (heterobeltosis) may be useful in identifying true heterotic cross combinations but these crosses can be immense practical value, if they show superiority over the standard or the best variety of the area. Realizing the importance of crop, the attempt has been made to estimate heterosis for seed yield and contributing characters.

\section{Materials and Methods}

The experimental material comprised of 57 entries, involving 39 crosses along with 16 parents and 2 checks viz., Chetak Aphim and JOP-540. The 39 crosses were generated by crossing 13 lines with 3 testers in $\mathrm{L} \times \mathrm{T}$ fashion during Rabi 2018-2019. The complete set of 57 genotypes were evaluated in randomized block design with 3 replication at Instructional farm, Rajasthan College of Agriculture, MPUAT, Udaipur during Rabi 2019-2020. The plot size for parent and hybrid comprised of one row each. Each row was 1.5 meter long with spacing of $30 \mathrm{~cm}$ between rows and $10 \mathrm{~cm}$ between plants was adopted. Five plants from each parent and hybrid were selected at random to record observations on eleven characters viz., days to 50 percent flowering, days to maturity, plant height $(\mathrm{cm})$, peduncle length $(\mathrm{cm})$, number of leaves per plant, number of stigmatic rays per capsule, diameter of main capsule (mm), capsule husk yield per plant (g), seed yield per plant (g), latex yield per plant (g) and harvest index for seed yield $(\%)$.

\section{Results and Discussion}

The analysis of variance revealed the presence of variability among parents and crosses for the characters studied. The parents $\mathrm{v} / \mathrm{s}$ hybrids were significant for all the characters, except for days to 50 percent flowering, days to maturity and number of leaves per plant, indicated the presence of considerable amount of genetic diversity in the material tested (Table 1-5).

\section{Days to 50 percent flowering}

The significant estimates of heterosis, heterobeltosis and economic heterosis were not expressed by any of the hybrids for days to 50 percent flowering.

\section{Days to maturity}

For days to maturity, UOP-125 x UOP-80 exhibited significant negative relative heterosis (-4.23) and heterobeltosis (-3.84), respectively. Three hybrids viz., UOP-68 x UOP-80 (-4.09), UOP-125 x UOP-80 (-4.36) and UOP-68 x UOP-79 (-4.63) exhibited significant negative economic heterosis over the best check Chetak Aphim.

\section{Plant height}

For this trait, the magnitude of negative significant relative heterosis and heterobeltosis ranged from -4.71 (UOP-125 x UOP-79) to -6.56 (UOP-30 x UOP-80) and 5.16 (UOP-70 x UOP-80) to 6.40 (UOP-125 x UOP-80), respectively. UOP-179 x UOP-79 (5.87) was the only hybrid which exhibited significant negative economic heterosis over best check Chetak Aphim.

\section{Peduncle length}

The positive significant relative heterosis for the trait was exhibited by six hybrids which ranged from 8.21 (UOP-71 $x$ UOP-80) to 31.91 (UOP-145 x UOP-20). Hybrids UOP37 x UOP-80 (12.30) and UOP-145 x UOP20 (23.74) exhibited positive significant heterobeltosis for the above character. The 
economic heterosis over best check JOP-540 was noticed in fourteen hybrids which ranged from 11.12 (UOP-128 $\mathrm{x}$ UOP-80) to 31.81(UOP-145 x UOP-20).

\section{Number of stigmatic rays per capsule}

Twenty five hybrids exhibited positive significant relative heterosis which ranged from 9.53 (UOP-64 x UOP-20) to 33.63 (UOP-64 X UOP-80). Significant heterobeltosis was expressed by nineteen hybrids which ranged from 9.64 (UOP-128 x UOP-20) to 29.41 (UOP-37 x UOP-79).

\section{Diameter of main capsule}

Hybrid UOP-71 x UOP-79 manifested significant positive relative heterosis (21.28) and heterobeltosis (18.35) for the character.

\section{Capsule husk yield per plant}

The estimates of relative heterosis was noticed in eight hybrids ranged which from 18.03 (UOP-71 x UOP-79) to 37.18 (UOP$149 \times$ UOP-79). UOP-30 x UOP-20 (26.74) and UOP-149 x UOP-79 (32.25) were the only two hybrids which exhibited positive significant heterobeltosis for husk yield per plant. UOP-149 x UOP-79 (31.00) was the only hybrid which expressed positive significant economic heterosis over the best check Chetak Aphim.

\section{Seed yield per plant}

The estimates of heterosis over mid parent was found positively significant for fifteen hybrids which ranged from 23.85 (UOP-93 x UOP-20) to 89.23 (UOP-128 x UOP-20. Significant positive heterobeltosis was observed in eleven hybrids which varied from 27.04 (UOP-35 x UOP-79) to 80.88 (UOP$128 \times$ UOP-20). The only hybrid UOP-128 x UOP-20 (23.39) exhibited positive significant economic heterosis over the best check Chetak Aphim.

Table.1 Analysis of variance for eleven traits in Opium poppy

\begin{tabular}{|l|r|r|r|r|r|r|}
\hline Characters & Rep & Genotype & Parents & Crosses & P Vs C & Error \\
\hline & {$[2]$} & {$[56]$} & {$[15]$} & {$[38]$} & {$[1]$} & {$[112]$} \\
\hline Days to 50 \% flowering & $37.02^{* *}$ & $10.73^{* *}$ & $12.48^{*}$ & $10.42^{*}$ & 3.81 & 6.26 \\
\hline Days to maturity & $46.33^{* *}$ & $8.89^{* *}$ & 6.53 & $10.11^{* *}$ & 6.85 & 4.50 \\
\hline Plant height & 1.82 & $47.73^{* *}$ & $51.25^{* *}$ & $47.34^{* *}$ & $24.53^{*}$ & 5.92 \\
\hline Peduncle length & $14.90^{* *}$ & $15.26^{* *}$ & $19.13^{* *}$ & $13.27 * *$ & $25.51 * *$ & 2.27 \\
\hline Number of leaves per plant & $0.79^{*}$ & $0.76^{* *}$ & $0.78^{* *}$ & $0.81^{* *}$ & 0.23 & 0.17 \\
\hline $\begin{array}{l}\text { Number of stigmatic rays per } \\
\text { capsule }\end{array}$ & $8.28^{* *}$ & $4.21^{* *}$ & $2.56^{* *}$ & $2.37 * *$ & $80.15^{* *}$ & 0.25 \\
\hline Diameter of main capsule & $81.38^{* *}$ & 14.41 & 11.41 & 14.50 & $51.90^{*}$ & 12.93 \\
\hline Capsule husk yield per plant & $1.53^{* *}$ & $0.50^{*}$ & 0.27 & 0.44 & $6.68^{* *}$ & 0.30 \\
\hline Latex yield per plant & $3.38^{*}$ & $5.82^{* *}$ & $4.11^{* *}$ & $5.57 * *$ & $43.27 * *$ & 0.87 \\
\hline Seed yield per plant & $0.00^{* *}$ & $0.00^{* *}$ & $0.00^{* *}$ & $0.00^{* *}$ & $0.03 * *$ & 0.00 \\
\hline Harvest index for seed yield & $82.67^{*}$ & $108.41^{* *}$ & $142.25^{* *}$ & $92.21^{* *}$ & $245.15^{* *}$ & 19.16 \\
\hline
\end{tabular}

$*$, ** Significant at $5 \%$ and $1 \%$ level of significance, respectively 
Table.2 Extent of heterosis for days to $50 \%$ flowering, days to maturity and plant height

\begin{tabular}{|c|c|c|c|c|c|c|c|c|c|}
\hline \multirow{2}{*}{ Crosses } & \multicolumn{3}{|c|}{ Days to $50 \%$ flowering } & \multicolumn{3}{|c|}{ Days to maturity } & \multicolumn{3}{|c|}{ Plant height } \\
\hline & Het & $\mathrm{Hb}$ & EH & Het & $\mathrm{Hb}$ & $\mathrm{EH}$ & Het & $\mathrm{Hb}$ & $\mathrm{EH}$ \\
\hline UOP-30 x UOP-20 & 0.95 & - & - & 1.92 & - & - & 1.65 & - & - \\
\hline UOP-35 x UOP-20 & -0.19 & - & -0.38 & 1.79 & - & - & $6.95 * *$ & - & - \\
\hline UOP-37 x UOP-20 & -0.19 & - & -1.89 & 0.28 & - & -1.09 & $-5.83 * *$ & $-5.30 *$ & - \\
\hline UOP-68 x UOP-20 & 3.83 & - & - & $2.64 *$ & - & - & $7.90 * *$ & - & - \\
\hline UOP-70 x UOP-20 & -1.15 & -0.77 & -2.27 & -0.28 & - & -2.18 & -0.49 & - & - \\
\hline UOP-71 x UOP-20 & -1.87 & 0.00 & -0.76 & -0.41 & 0.00 & -0.82 & -3.44 & -3.18 & - \\
\hline UOP-93 x UOP-20 & 2.80 & - & - & 0.82 & - & - & 2.76 & - & - \\
\hline UOP-125 x UOP-20 & 2.63 & - & - & 0.00 & - & -0.27 & $-4.87 * *$ & -3.68 & - \\
\hline UOP-128 x UOP-20 & 1.15 & - & - & 0.55 & - & -0.54 & $7.74 * *$ & - & - \\
\hline UOP-145 x UOP-20 & 0.57 & - & 0.00 & -0.27 & -0.27 & -1.09 & 3.40 & - & - \\
\hline UOP-149 x UOP-20 & 1.91 & - & - & -0.55 & -0.28 & -1.63 & $-5.88 * *$ & -3.29 & -2.91 \\
\hline UOP-179 x UOP-20 & -1.14 & -0.38 & -1.14 & -0.96 & -0.82 & -1.63 & -2.03 & - & -0.49 \\
\hline UOP-30 x UOP-79 & 2.48 & - & - & 1.24 & - & 0.00 & $5.66 * *$ & - & - \\
\hline UOP-35 x UOP-79 & -0.19 & - & -1.14 & 0.83 & - & -0.82 & $4.43 *$ & - & - \\
\hline UOP-37 x UOP-79 & 1.75 & - & -0.76 & 1.81 & - & -0.27 & $-6.22 * *$ & -4.17 & -1.64 \\
\hline UOP-64 x UOP-79 & -0.74 & - & - & 0.96 & - & - & $4.97 * *$ & - & - \\
\hline UOP-68 x UOP-79 & -1.16 & -0.78 & -3.03 & -1.96 & -1.41 & $-4.63 * *$ & 1.92 & - & - \\
\hline UOP-70 x UOP-79 & 1.16 & - & -0.76 & $2.66^{*}$ & - & 0.00 & -2.73 & - & - \\
\hline UOP-125 x UOP-79 & -0.38 & - & -0.38 & -0.96 & - & -1.91 & $-4.71 *$ & -1.95 & - \\
\hline UOP-128 x UOP-79 & $5.00 *$ & - & - & 1.80 & - & 0.00 & $9.41 * *$ & - & - \\
\hline UOP-145 x UOP-79 & 2.11 & - & - & 0.69 & - & -0.82 & 2.41 & - & - \\
\hline UOP-149 x UOP-79 & $5.38^{* *}$ & - & - & $2.91 *$ & - & - & 0.22 & - & - \\
\hline UOP-179 x UOP-79 & 1.53 & - & - & 0.00 & - & -1.36 & $-5.80 * *$ & -3.18 & $-5.87 *$ \\
\hline UOP-30 x UOP-80 & -1.88 & -1.51 & -1.14 & -0.96 & -0.82 & -1.36 & $-6.56 * *$ & $-6.15 * *$ & - \\
\hline UOP-35 x UOP-80 & -0.75 & -0.75 & -0.38 & 0.82 & - & 0.00 & -0.57 & - & - \\
\hline UOP-37 x UOP-80 & 1.53 & - & - & 0.69 & - & -0.54 & -0.33 & - & - \\
\hline UOP-64 x UOP-80 & 0.18 & - & - & 0.68 & - & - & 0.84 & - & - \\
\hline UOP-68 x UOP-80 & -1.71 & -0.77 & -2.27 & -2.22 & -0.85 & $-4.09 * *$ & 2.33 & - & - \\
\hline UOP-70 x UOP-80 & 3.24 & - & - & $2.64^{*}$ & - & - & $-5.80 * *$ & $-5.16^{*}$ & - \\
\hline UOP-71 x UOP-80 & 0.56 & - & - & 0.00 & - & -0.27 & $5.63 * *$ & - & - \\
\hline UOP-93 x UOP-80 & 2.97 & - & - & 1.50 & - & - & -2.91 & -1.50 & - \\
\hline UOP-125 x UOP-80 & -3.93 & -3.02 & -2.65 & $-4.23 * *$ & $-3.84 * *$ & $-4.36 * *$ & $-6.54 * *$ & $-6.40 * *$ & - \\
\hline UOP-128 x UOP-80 & 3.23 & - & - & 0.14 & - & -0.82 & $5.44 * *$ & - & - \\
\hline UOP-145 x UOP-80 & 3.41 & - & - & 1.23 & - & - & 3.24 & - & - \\
\hline UOP-149 x UOP-80 & 2.09 & - & - & 0.41 & - & -0.54 & 2.72 & - & - \\
\hline UOP-179 x UOP-80 & 2.45 & - & - & 1.10 & - & - & 2.37 & - & - \\
\hline
\end{tabular}

*, ** Significant at $5 \%$ and $1 \%$ level of significance, respectively. 
Table.3 Extent of heterosis for peduncle length, number of leaves per plant and number of stigmatic rays per capsule

\begin{tabular}{|c|c|c|c|c|c|c|c|c|c|}
\hline \multirow[t]{2}{*}{ Crosses } & \multicolumn{3}{|c|}{ Peduncle length } & \multicolumn{3}{|c|}{ Number of leaves per plant } & \multicolumn{3}{|c|}{ Number of stigmatic rays per capsule } \\
\hline & Het & $\mathrm{Hb}$ & EH & Het & $\mathrm{Hb}$ & EH & Het & $\mathrm{Hb}$ & $\mathrm{EH}$ \\
\hline UOP-30 x UOP-20 & -3.67 & - & 6.52 & 2.28 & 0.00 & 1.51 & 4.80 & 4.49 & - \\
\hline UOP-35 x UOP-20 & 1.82 & - & $21.88^{* *}$ & $6.75^{*}$ & 2.89 & $7.55 *$ & 2.23 & 0.99 & - \\
\hline UOP-37 x UOP-20 & -2.91 & - & 2.51 & $-5.43 *$ & - & - & 4.48 & 0.39 & - \\
\hline UOP-64 x UOP-20 & -7.68 & - & - & $-6.93 *$ & - & - & $9.53 *$ & - & - \\
\hline UOP-68 x UOP-20 & 1.89 & - & $13.55^{*}$ & 1.02 & - & 4.53 & $23.71^{* *}$ & $15.51^{* * *}$ & - \\
\hline UOP-70 x UOP-20 & $-8.76^{*}$ & - & 1.88 & -2.63 & - & - & -4.23 & - & - \\
\hline UOP-71 x UOP-20 & $8.73^{*}$ & 0.35 & $26.38^{* *}$ & 2.71 & 0.31 & - & $18.64^{* *}$ & 5.42 & - \\
\hline UOP-93 x UOP-20 & $-14.23^{* *}$ & - & - & -1.62 & - & 0.60 & $25.00^{* *}$ & $17.47^{* *}$ & - \\
\hline UOP-125 x UOP-20 & 1.93 & - & $18.41 * *$ & $8.81 * *$ & $7.98^{*}$ & $6.34 *$ & 2.41 & 2.41 & - \\
\hline UOP-128 x UOP-20 & -1.31 & - & 3.33 & $-6.69 *$ & - & - & $11.09^{* *}$ & $9.64 *$ & - \\
\hline UOP-145 x UOP-20 & $31.91 * *$ & $23.74 * *$ & $31.81 * *$ & 2.35 & 1.56 & - & 12.31 ** & $11.98^{* *}$ & - \\
\hline UOP-149 x UOP-20 & -6.21 & - & 7.25 & 1.55 & 0.92 & - & 6.30 & 5.36 & - \\
\hline UOP-179 x UOP-20 & -3.06 & - & 1.16 & $6.48 *$ & 4.98 & 1.81 & $19.97 * *$ & $14.91 * *$ & - \\
\hline UOP-30 x UOP-79 & $-9.30^{*}$ & - & 0.87 & -0.30 & - & 0.30 & $11.95^{* *}$ & 6.59 & - \\
\hline UOP-35 x UOP-79 & $-15.06^{* *}$ & - & 2.22 & $-6.89 * *$ & - & - & -3.10 & - & - \\
\hline UOP-37 x UOP-79 & $-14.44 * *$ & - & - & $-7.34 * *$ & - & - & $30.26^{* *}$ & $29.41 * *$ & - \\
\hline UOP-64 x UOP-79 & -6.16 & - & - & -2.13 & - & - & $26.46^{* *}$ & $16.56^{* * *}$ & - \\
\hline UOP-68 x UOP-79 & -6.10 & - & 5.25 & $-12.97 * *$ & - & - & $12.64 * *$ & $10.03 *$ & - \\
\hline UOP-70 x UOP-79 & 4.92 & 0.87 & $17.83 * *$ & -4.88 & - & - & $22.60^{* *}$ & $18.54 * *$ & - \\
\hline UOP-71 x UOP-79 & $-8.85 *$ & - & 6.52 & 5.03 & 1.21 & 0.91 & 14.29 ** & 5.96 & - \\
\hline UOP-93 x UOP-79 & $-11.74 * *$ & - & - & -0.87 & - & 2.72 & $19.19^{* *}$ & $17.22 * *$ & - \\
\hline UOP-125 x UOP-79 & -6.36 & - & 9.36 & $-7.93 * *$ & - & - & $9.46^{* *}$ & 4.52 & - \\
\hline UOP-128 x UOP-79 & 2.40 & 0.08 & 7.87 & 0.61 & - & - & 15.37 ** & $11.57 * *$ & - \\
\hline UOP-145 x UOP-79 & $13.57 * *$ & 5.96 & $14.20 * *$ & 4.12 & 1.91 & 1.60 & $22.11^{* *}$ & $16.26^{* *}$ & - \\
\hline UOP-149 x UOP-79 & -2.94 & - & $11.59^{*}$ & 2.60 & 1.82 & 1.51 & $22.19^{* *}$ & $15.68^{* * *}$ & - \\
\hline UOP-179 x UOP-79 & -4.05 & - & 0.72 & 4.36 & 1.52 & 1.21 & $19.14 * *$ & $18.75^{* * *}$ & - \\
\hline UOP-30 x UOP-80 & 5.78 & 1.77 & $16.67 * *$ & $-8.95 * *$ & - & - & 0.60 & 0.00 & - \\
\hline UOP-35 x UOP-80 & $-10.80^{* *}$ & - & 6.52 & -4.63 & - & - & 2.57 & 0.44 & - \\
\hline UOP-37 x UOP-80 & $12.99^{* *}$ & $12.30 *$ & $18.97^{* *}$ & 1.39 & 1.23 & - & $13.04 * *$ & $7.69^{*}$ & - \\
\hline UOP-64 x UOP-80 & -1.41 & - & 1.45 & 1.78 & 1.01 & 0.09 & $33.63^{* *}$ & $17.16^{* *}$ & - \\
\hline UOP-68 x UOP-80 & $-8.67 *$ & - & 1.52 & $-5.39 *$ & - & - & 5.59 & - & - \\
\hline UOP-70 x UOP-80 & 1.50 & - & $13.04 *$ & -1.48 & - & - & 5.58 & - & - \\
\hline UOP-71 x UOP-80 & $8.21 *$ & - & $25.46 * *$ & $11.07 * *$ & $8.14 *$ & 5.53 & $19.46^{* *}$ & 5.33 & - \\
\hline UOP-93 x UOP-80 & -1.24 & - & 9.86 & -3.98 & - & - & 21.90 ** & $13.61 * *$ & - \\
\hline UOP-125 x UOP-80 & $-11.57 * *$ & - & 2.46 & -2.62 & - & - & 0.30 & - & - \\
\hline UOP-128 x UOP-80 & 6.41 & 4.88 & $11.12 *$ & 0.87 & 0.71 & - & 5.25 & 2.96 & - \\
\hline UOP-145 x UOP-80 & $9.96 *$ & 3.42 & 9.57 & 4.85 & 3.72 & 1.21 & -0.39 & - & - \\
\hline UOP-149 x UOP-80 & 0.64 & - & $14.78 * *$ & $6.57 *$ & $6.25 *$ & 4.32 & $15.38^{* *}$ & $15.38^{* * *}$ & - \\
\hline UOP-179 x UOP-80 & 1.95 & 0.14 & 6.09 & $10.87 * *$ & $8.98 * *$ & $6.34 *$ & $26.48^{* *}$ & $20.12 * *$ & - \\
\hline
\end{tabular}

*,** Significant at $5 \%$ and $1 \%$ level of significance, respectively. 
Table.4 Extent of heterosis for diameter of main capsule, capsule husk yield per plant and seed yield per plant

\begin{tabular}{|c|c|c|c|c|c|c|c|c|c|}
\hline \multirow[t]{2}{*}{ Crosses } & \multicolumn{3}{|c|}{ Diameter of main capsule } & \multicolumn{3}{|c|}{ Capsule husk yield per plant } & \multicolumn{3}{|c|}{ Seed yield per plant } \\
\hline & Het & $\mathrm{Hb}$ & $\mathrm{EH}$ & Het & $\mathrm{Hb}$ & EH & Het & $\mathrm{Hb}$ & $\mathrm{EH}$ \\
\hline UOP-30 x UOP-20 & -0.37 & - & - & $27.38 * *$ & $26.74 *$ & 11.23 & 14.82 & 14.12 & - \\
\hline UOP-35 x UOP-20 & 5.73 & 5.51 & - & $18.47^{*}$ & 16.32 & 5.92 & 13.70 & 11.98 & - \\
\hline UOP-37 x UOP-20 & -2.61 & - & - & $21.71 *$ & 20.08 & 5.38 & $47.56^{* *}$ & $37.48 * *$ & 8.63 \\
\hline UOP-64 x UOP-20 & 12.29 & 12.27 & 0.40 & 14.81 & 11.11 & 4.24 & $54.75 * *$ & $50.88^{* *}$ & 2.93 \\
\hline UOP-68 x UOP-20 & 8.05 & 6.59 & - & 14.34 & 9.38 & 5.11 & 65.46 ** & $63.32 * *$ & 14.37 \\
\hline UOP-70 x UOP-20 & -7.06 & - & - & 6.95 & 0.00 & 0.87 & 8.22 & 5.85 & - \\
\hline UOP-71 x UOP-20 & 0.23 & - & - & 4.36 & 4.28 & - & -5.07 & - & - \\
\hline UOP-93 x UOP-20 & 2.29 & - & - & 3.59 & 0.14 & - & $23.85^{*}$ & 22.96 & - \\
\hline UOP-125 x UOP-20 & 2.09 & - & - & $20.48 *$ & 18.43 & 7.60 & 12.72 & - & - \\
\hline UOP-128 x UOP-20 & 11.93 & 11.85 & - & $18.74 *$ & 14.71 & 8.00 & $89.23 * *$ & $80.88^{* * *}$ & 23.39 \\
\hline UOP-145 x UOP-20 & 5.94 & 1.54 & - & 7.14 & 0.33 & 0.87 & $-19.33 *$ & - & - \\
\hline UOP-149 x UOP-20 & 8.16 & 4.43 & - & 14.83 & 8.28 & 7.26 & -0.30 & - & - \\
\hline UOP-179 x UOP-20 & -0.36 & - & - & 10.38 & 6.16 & 0.87 & $43.27 * *$ & $34.12 * *$ & 4.90 \\
\hline UOP-30 x UOP-79 & 10.03 & 9.70 & - & 12.82 & 9.73 & 0.87 & $35.40 * *$ & $29.34 *$ & - \\
\hline UOP-35 x UOP-79 & 5.80 & 5.62 & - & 12.68 & 12.14 & 3.09 & 34.16 ** & $27.04 *$ & - \\
\hline UOP-37 x UOP-79 & -4.09 & - & - & 13.77 & 9.73 & 0.87 & 15.95 & 4.11 & - \\
\hline UOP-64 x UOP-79 & -5.08 & - & - & 11.88 & 10.75 & 3.90 & $37.77 * *$ & $35.73^{*}$ & - \\
\hline UOP-68 x UOP-79 & 6.33 & 5.29 & - & 10.52 & 8.12 & 3.90 & -0.54 & - & - \\
\hline UOP-70 x UOP-79 & 3.50 & 2.23 & - & 2.90 & - & - & 22.57 & 15.30 & - \\
\hline UOP-71 x UOP-79 & $21.28 * *$ & $18.35^{*}$ & 6.60 & $18.03 *$ & 15.44 & 6.12 & $47.24 * *$ & $46.59 * *$ & - \\
\hline UOP-93 x UOP-79 & 5.89 & 2.16 & - & 4.16 & 3.00 & - & $49.21 * *$ & $42.38 * *$ & - \\
\hline UOP-125 x UOP-79 & 3.60 & 0.75 & - & 12.80 & 12.14 & 3.09 & $30.41 *$ & 14.81 & - \\
\hline UOP-128 x UOP-79 & -1.30 & - & - & 6.76 & 5.50 & - & 6.13 & 5.55 & - \\
\hline UOP-145 x UOP-79 & 5.39 & 1.39 & - & 8.04 & 3.41 & 3.97 & $28.12 * *$ & 5.92 & 1.93 \\
\hline UOP-149 x UOP-79 & 12.44 & 8.17 & - & $37.18^{* * *}$ & $32.25^{* *}$ & $31.00 * *$ & $71.00^{* *}$ & $58.77 * *$ & - \\
\hline UOP-179 x UOP-79 & 7.53 & 2.72 & 1.61 & 10.29 & 8.49 & 3.09 & 14.62 & 3.39 & - \\
\hline UOP-30 x UOP-80 & -3.15 & - & - & 15.44 & 4.04 & 12.64 & 1.96 & - & - \\
\hline UOP-35 x UOP-80 & -8.54 & - & - & 5.13 & - & 4.77 & -0.02 & - & - \\
\hline UOP-37 x UOP-80 & 1.22 & - & - & $16.87^{*}$ & 4.53 & 13.18 & 10.47 & - & 1.40 \\
\hline UOP-64 x UOP-80 & -0.17 & - & - & -0.17 & - & 0.87 & $-19.17^{*}$ & - & - \\
\hline UOP-68 x UOP-80 & 1.05 & - & - & 10.50 & 4.29 & 12.91 & $-20.62 *$ & - & - \\
\hline UOP-70 x UOP-80 & -0.18 & - & - & $-16.08 *$ & - & - & -16.10 & - & - \\
\hline UOP-71 x UOP-80 & 12.26 & 4.03 & 4.49 & 6.68 & - & 4.64 & $-32.92 * *$ & - & - \\
\hline UOP-93 x UOP-80 & -8.97 & - & - & -9.91 & - & - & 4.73 & - & - \\
\hline UOP-125 x UOP-80 & -9.15 & - & - & 1.32 & - & 0.87 & $37.60 * *$ & 0.27 & 4.86 \\
\hline UOP-128 x UOP-80 & -0.34 & - & - & -6.98 & - & - & $-19.63 *$ & - & - \\
\hline UOP-145 x UOP-80 & -2.62 & - & - & -3.38 & - & 0.87 & $-16.75^{*}$ & - & - \\
\hline UOP-149 x UOP-80 & 4.16 & - & - & 0.55 & - & 4.24 & 14.61 & - & - \\
\hline UOP-179 x UOP-80 & 3.45 & 2.66 & 3.12 & -14.26 & - & - & $-27.99 * *$ & - & - \\
\hline
\end{tabular}

*, ** Significant at $5 \%$ and $1 \%$ level of significance, respectively. 
Table.5 Extent of heterosis for latex yield per plant and harvest index for seed yield

\begin{tabular}{|c|c|c|c|c|c|c|}
\hline \multirow[t]{2}{*}{ Crosses } & \multicolumn{3}{|c|}{ Latex yield per plant } & \multicolumn{3}{|c|}{ Harvest index for seed yield } \\
\hline & Het & $\mathrm{Hb}$ & EH & Het & $\mathrm{Hb}$ & EH \\
\hline UOP-30 x UOP-20 & $32.32 *$ & 10.45 & - & 4.14 & 2.50 & - \\
\hline UOP-35 x UOP-20 & 26.19 & 16.38 & - & 4.62 & - & - \\
\hline UOP-37 x UOP-20 & $35.58^{*}$ & 8.19 & - & 11.50 & 2.07 & 1.49 \\
\hline UOP-64 x UOP-20 & $44.51 * *$ & $33.90 *$ & 1.07 & $17.39 * *$ & $14.65 *$ & - \\
\hline UOP-68 x UOP-20 & 17.41 & - & - & $31.66 * *$ & $23.31 * *$ & 1.88 \\
\hline UOP-70 x UOP-20 & $62.45^{* *}$ & 27.12 & - & 3.13 & - & - \\
\hline UOP-71 x UOP-20 & -10.33 & - & - & $-15.65^{*}$ & - & - \\
\hline UOP-93 x UOP-20 & 9.60 & 7.95 & - & 5.80 & - & - \\
\hline UOP-125 x UOP-20 & $34.12 *$ & 12.71 & - & -0.21 & - & - \\
\hline UOP-128 x UOP-20 & -11.88 & - & - & $18.47 * *$ & $14.28^{*}$ & 1.60 \\
\hline UOP-145 x UOP-20 & $31.02 *$ & 17.51 & - & $-20.51 * *$ & - & - \\
\hline UOP-149 x UOP-20 & 1.68 & - & - & 7.47 & - & - \\
\hline UOP-179 x UOP-20 & -17.42 & - & - & $13.42 *$ & 10.25 & - \\
\hline UOP-30 x UOP-79 & $34.51^{*}$ & 8.55 & - & $14.03^{*}$ & 9.43 & - \\
\hline UOP-35 x UOP-79 & -9.78 & - & - & $31.80 * *$ & $31.16^{* *}$ & - \\
\hline UOP-37 x UOP-79 & $35.34 *$ & 4.66 & - & 11.65 & - & - \\
\hline UOP-64 x UOP-79 & 8.43 & - & - & $23.12 * *$ & 13.80 & - \\
\hline UOP-68 x UOP-79 & 20.39 & - & - & 12.55 & 11.48 & - \\
\hline UOP-70 x UOP-79 & $48.46 * *$ & 12.69 & - & 9.91 & - & - \\
\hline UOP-71 x UOP-79 & 15.68 & - & - & $15.72 *$ & 5.06 & - \\
\hline UOP-93 x UOP-79 & -17.18 & - & - & $17.89 * *$ & 5.37 & - \\
\hline UOP-125 x UOP-79 & 3.03 & - & - & $30.29 * *$ & $24.37 * *$ & - \\
\hline UOP-128 x UOP-79 & 27.57 & 1.30 & - & 2.12 & - & - \\
\hline UOP-145 x UOP-79 & -1.05 & - & - & 4.95 & - & - \\
\hline UOP-149 x UOP-79 & 8.60 & - & - & $43.66^{* *}$ & $35.18 * *$ & - \\
\hline UOP-179 x UOP-79 & 16.91 & 5.70 & - & $13.73^{*}$ & 4.66 & - \\
\hline UOP-30 x UOP-80 & 25.75 & 0.51 & - & 6.96 & - & - \\
\hline UOP-35 x UOP-80 & 17.41 & 3.03 & - & 10.89 & - & - \\
\hline UOP-37 x UOP-80 & $64.42 * *$ & 26.01 & 6.40 & -0.33 & - & - \\
\hline UOP-64 x UOP-80 & 1.43 & - & - & $-17.14 * *$ & - & - \\
\hline UOP-68 x UOP-80 & 18.79 & - & - & $-13.59^{*}$ & - & - \\
\hline UOP-70 x UOP-80 & $31.88^{*}$ & - & - & $-16.32 * *$ & - & - \\
\hline UOP-71 x UOP-80 & 4.45 & - & - & $-29.71 * *$ & - & - \\
\hline UOP-93 x UOP-80 & -11.43 & - & - & -2.90 & - & - \\
\hline UOP-125 x UOP-80 & 16.80 & - & - & $19.37 * *$ & 0.01 & - \\
\hline UOP-128 x UOP-80 & $30.98^{*}$ & 3.03 & - & -9.72 & - & - \\
\hline UOP-145 x UOP-80 & 4.87 & - & - & $-14.47 * *$ & - & - \\
\hline UOP-149 x UOP-80 & 17.24 & - & - & $17.81 * *$ & - & - \\
\hline UOP-179 x UOP-80 & 18.36 & 5.81 & - & $-13.01^{*}$ & - & - \\
\hline
\end{tabular}

*, ** Significant at $5 \%$ and $1 \%$ level of significance, respectively. 


\section{Latex yield per plant}

Twelve hybrids manifested significant positive relative heterosis which ranged from 30.98 (UOP-128 x UOP-80) to 64.42 (UOP37 x UOP-80). Hybrid UOP-64 x UOP-20 (33.9) exhibited positive significant heterobeltosis for latex yield per plant.

\section{Harvest index for seed yield}

Fourteen hybrids exhibited significant positive heterosis over mid parent which ranged from 13.42 (UOP-179 x UOP-20) to 43.66 (UOP-149 x UOP-79). Significant positive heterobeltosis was observed in six hybrids which ranged from 14.28 (UOP-128 x UOP-20) to 35.18 (UOP-149 x UOP-79). Similar results on economic heterosis, heterobeltosis and relative heterosis in opium poppy for seed yield and yield contributing traits were reported by Patidar (1994), Yadav et al., (2007), Dubey et al., (2007), Kumar et al., (2008) and Singh and Pandey (2011).

Thus, in the present study, cross UOP-128 $\times$ UOP-20 found promising with respect to economic heterosis which can be handled according to some breeding method.

\section{References}

Dubey, R.B., Jain, S.K. and Maloo, S.R.
2007. Combining Ability and Heterosis for Latex Yield, Seed Yield and other Agronomic Traits in Opium Poppy (Papaver somniferum L.). The Indian Journal of Genetics and Plant Breeding, 67(4): 392-395.

Kumar, B., Singh, H.P., Verma, A.K., Misra, H.O. and Patra, N.K. 2008. Combining ability analysis in relation to heterosis in Opium poppy (Papaver somniferum L.). J. Medicinal and Aromatic Plant Sci., 30: 83-87.

Patidar, H. 1994. Hybrid vigour in Opium Poppy. Indian. Genet., 54(4): 395-397.

Singh, R. and Pandey, R.M. 2011. Combining Ability and Heterosis in Opium Poppy (Papaver somniferum L.). Current Advances in Agricultural Sciences, 3(2): 130-134.

Singh, S.P., Shukla, S. and Khanna, K.R. 1999. Breeding strategies in opium poppy (Papaver somniferum L.) at National Botanical Research Institute, Lucknow, India. Applied Botany Abstracts, 19(2): 121-39.

Yadav, H.K., Singh, S. and Singh, S.P. 2007. Genetic divergence in parental genotypes and its relation with heterosis, $\mathrm{F}_{1}$ performance and general combining ability (GCA) in opium poppy (Papaver somniferum L.). Euphytica, 157: 123-130.

\section{How to cite this article:}

Nesara, K., Amit Dadheech, N.K. Padiwal, S. Bhaskar Reddy and Rajwanti Saran. 2020. Estimates of Heterosis for Seed Yield and its Contributing Characters in Opium Poppy (Papaver somniferum L.). Int.J.Curr.Microbiol.App.Sci. 9(09): 2566-2573. doi: https://doi.org/10.20546/ijcmas.2020.909.321 